\title{
An FBSDE Approach to American Option Pricing with an Interacting Particle Method*
}

\author{
Masaaki Fujii ${ }^{\dagger}$ Seisho Sato ${ }^{\ddagger}$ Akihiko Takahashi ${ }^{\S}$
}

First version: November 26, 2012

\begin{abstract}
In the paper, we propose a new calculation scheme for American options in the framework of a forward backward stochastic differential equation (FBSDE). The wellknown decomposition of an American option price with that of a European option of the same maturity and the remaining early exercise premium can be cast into the form of a decoupled non-linear FBSDE. We numerically solve the FBSDE by applying an interacting particle method recently proposed by Fujii \& Takahashi (2012d), which allows one to perform a Monte Carlo simulation in a fully forward-looking manner. We perform the fourth-order analysis for the Black-Scholes (BS) model and the thirdorder analysis for the Heston model. The comparison to those obtained from existing tree algorithms shows the effectiveness of the particle method.
\end{abstract}

Keywords : BSDE, FBSDE, asymptotic expansion, perturbation, particle method

\footnotetext{
*This research is supported by CARF (Center for Advanced Research in Finance) and the global COE program "The research and training center for new development in mathematics." All the contents expressed in this research are solely those of the authors and do not represent any views or opinions of any institutions. The authors are not responsible or liable in any manner for any losses and/or damages caused by the use of any contents in this research.

${ }^{\dagger}$ Graduate School of Economics, The University of Tokyo

$\ddagger_{\text {The Institute of Statistical Mathematics }}$

${ }^{\S}$ Graduate School of Economics, The University of Tokyo
} 


\section{Introduction}

It has been known for a while that an American option value can be decomposed into that of the corresponding European option and an additional early exercise premium. Detailed discussions and other related references are available in Kim (1990) [24], Carr et.al. (1992) [7], Jacka (1991) [21, Rutkowski (1994) [32, Saito \& Takahashi (2003) [33] as well as a textbook written by Karatzas \& Shreve (1998) [23. See also a recent work of Benth (2003) [2] which derives it from a dynamic programming approach. In this paper, we deal with a non-linear forward backward stochastic differential equation (FBSDE) obtained from the decomposition formula and calculate an American option price by solving it numerically.

The framework of FBSDE was first introduced by Bismut (1973) [5], and then later extended by Pardoux and Peng (1990) 31] for general non-linear cases. Their financial applications are discussed in details in, for example, El Karoui, Peng and Quenez (1997) [11] and Ma and Yong (2000) [28]. There are increasing interests among researchers in FBSDEs since their relevance for the analysis of various social phenomena is becoming more apparent in recent years. In fact, one can find FBSDEs in the valuation problem of the financial contracts in the presence of credit risk and/or funding cost of collaterals (Duffie \& Huang (1996) [10], Fujii \& Takahashi (2012a) [13, Crépey (2012) [8], for examples. ). They are also relevant for the utility-indifference pricing in incomplete as well as constrained markets ( Carmona (2009) [6] and references therein. ). In a recent book of Cvitanić and Zhang (2012) 9], the authors use FBSDEs to study the optimal contract theory in continuous time.

Recently, Fujii \& Takahashi (2012b) [14] has proposed a perturbative technique for generic non-linear FBSDEs. With the help of asymptotic expansion ( Takahashi (1999) 34] ), it is possible to derive closed-form analytic expressions for both of the backward components. An explicit example for a quadratic-growth FBSDE appearing in the optimal portfolio problem in an incomplete market is available in Fujii \& Takahashi (2012c) [15]. In the following paper, Fujii \& Takahashi (2012d) [16] gave its numerical evaluation scheme based on an interacting particle method inspired by the work of McKean (1975) [29], 1 ] which enables one to perform Monte Carlo simulation in a fully forward-looking manner. 2 The validity of its approximation is discussed recently by Takahashi \& Yamada (2012b) [37] although it is still restricted to a decoupled non-linear setup. In the current paper, we apply this methodology to evaluate a non-linear FBSDE relevant for an American option. Although there remains a small error when the option is far in the money, we shall see the effectiveness of the particle method in overall region. The current work not only gives a simple calculation scheme for American options but also serves as a concrete example showing the usefulness of the particle method to analyze non-linear FBSDEs and corresponding non-linear partial differential equations.

\footnotetext{
${ }^{1}$ It is closely related to the research with a long history on the branching Markov process and a certain class of semi-linear PDEs. For instance, see Fujita(1966) [12], Ikeda, Nagasawa \& Watanabe(1965,1966,1968) [17, [1], [19], Ikeda et.al.(1966,1967) [20] and Nagasawa \& Sirao (1969) 30.

${ }^{2}$ A related but different approach was recently applied to evaluate CVA by Henry-Labordère (2012) [26].
} 


\section{FBSDE formulation}

Let us take the probability space as $(\Omega, \mathcal{F}, \mathbb{Q})$, where $\mathbb{Q}$ is a risk-neutral probability measure. We consider a generic process for the relevant stock price as

$$
d S_{t}=\left(r_{t}-y_{t}\right) S_{t} d t+S_{t} \sigma_{t} \cdot d W_{t},
$$

where $W$ is a $d$-dimensional $\mathbb{Q}$-Brownian motion and $\mathcal{F}$ is a natural filtration generated by $W$. All the stochastic processes are assumed to be $\mathcal{F}_{t}$-adapted. Here, $r$ and $y$ are processes for a risk-free interest rate and a dividend yield, respectively. $\sigma \in \mathbb{R}^{d}$ is a volatility process.

It is well-known (e.g. [24, 7, 21, 32, 23]) that the price of an American option on $S$ with a strike $K$ and an expiry $T$ can be expressed as

$$
V_{t}=\beta_{t} \mathbb{E}\left[\beta_{T}^{-1} \Psi^{+}\left(S_{T}\right) \mid \mathcal{F}_{t}\right]+\beta_{t} \mathbb{E}\left[\int_{t}^{T} \beta_{u}^{-1} C_{u} \mathbf{1}_{\left\{V_{u} \leq \Psi^{+}\left(S_{u}\right)\right\}} d u \mid \mathcal{F}_{t}\right],
$$

where $\Psi^{+}(x)=\max (\Psi(x), 0)$ denotes a payoff function, which is

$$
\Psi(x)=\left\{\begin{array}{ll}
x-K & \text { for a Call } \\
K-x & \text { for a Put }
\end{array} .\right.
$$

$C_{t}$ is a process denoting an instantaneous early exercise premium

$$
C_{t}= \begin{cases}y_{t} S_{t}-r_{t} K & \text { for a Call } \\ r_{t} K-y_{t} S_{t} & \text { for a Put }\end{cases}
$$

and

$$
\beta_{t}=\exp \left(\int_{0}^{t} r_{s} d s\right)
$$

is a standard money-market account.

In the remaining part of this section, we provide a simple heuristic derivation of Eq. (2.2) for completeness. Firstly, let us provide the decomposition principle of the Snell envelope for a continuous semimartingale.

Proposition 1 Rutokowski (1994) [32]

Suppose $X$ is a continuous semimartingale with canonical decomposition

$$
X=X_{0}+M+V
$$

where $X_{0}$ is a constant, $M$ is a continuous local martingale with $M_{0}=0$, and $V$ denotes a continuous finite variation process with $V_{0}=0$, whose decreasing component satisfies $d V_{t}^{d}=\nu_{t} d t$ for some adapted nonnegative process $\nu$. We assume that the condition

$$
\mathbb{E}\left[\sup _{0 \leq t \leq T}\left|X_{t}\right|\right]<\infty
$$

is satisfied. Let $\left\{\tau_{t}^{*}\right\}_{t \in[0, T]}$ be a family of $\left\{\mathcal{F}_{t}\right\}$-stopping times satisfying

$$
\mathbb{E}\left[X_{\tau_{t}^{*}}\right]=\operatorname{ess} \sup _{\mathrm{t} \leq \tau \leq \mathrm{T}} \mathbb{E}\left[\mathrm{X}_{\tau} \mid \mathcal{F}_{\mathrm{t}}\right], \quad \forall \mathrm{t} \in[0, \mathrm{~T}] .
$$


Then the following equation holds:

$$
\mathbb{E}\left[X_{\tau_{t}^{*}} \mid \mathcal{F}_{t}\right]=\mathbb{E}\left[X_{T} \mid \mathcal{F}_{t}\right]-\mathbb{E}\left[\int_{\tau_{t}^{*}}^{T} \mathbf{1}_{\left\{\tau_{u}^{*}=u\right\}} d V_{u} \mid \mathcal{F}_{t}\right] .
$$

Proof: See Appendix of [32].

For concreteness, let us choose a Call option with strike $K$ as an example. We consider the dynamics of the discounted payoff process. By applying Itô formula, we obtain

$$
\begin{aligned}
d\left(\beta_{t}^{-1}\left(S_{t}-K\right)^{+}\right)= & -\beta_{t}^{-1} r_{t}\left(S_{t}-K\right)^{+} d t+\beta_{t}^{-1}\left\{\mathbf{1}_{\left\{S_{t} \geq K\right\}} d S_{t}+\frac{1}{2} \delta\left(X_{t}-K\right) d\langle S\rangle_{t}\right\} \\
= & \beta_{t}^{-1} \mathbf{1}_{\left\{S_{t} \geq K\right\}} S_{t} \sigma_{t} \cdot d W_{t} \\
& +\beta_{t}^{-1} \mathbf{1}_{\left\{S_{t} \geq K\right\}}\left(r_{t} K-y_{t} S_{t}\right) d t+\frac{1}{2} \beta_{t}^{-1} \delta\left(S_{t}-K\right) d\langle S\rangle_{t},
\end{aligned}
$$

where $\delta(\cdot)$ is a Dirac delta function. More precisely speaking, the term involves the delta function is represented by the local time. For our intuitive derivation, however, the Dirac delta function is more useful to borrow a clear economic insight in a later stage. Now, applying Proposition 1 gives

$$
\begin{aligned}
V_{t}= & \operatorname{ess} \sup _{\mathrm{t} \leq \tau \leq \mathrm{T}} \beta_{\mathrm{t}} \mathbb{E}\left[\beta_{\tau}^{-1}\left(\mathrm{~S}_{\tau}-\mathrm{K}\right)^{+} \mid \mathcal{F}_{\mathrm{t}}\right] \\
= & \beta_{t} \mathbb{E}\left[\beta_{T}^{-1}\left(S_{T}-K\right)^{+} \mid \mathcal{F}_{t}\right] \\
& +\beta_{t} \mathbb{E}\left[\int_{\tau_{t}^{*}}^{T} \mathbf{1}_{\left\{\tau_{u}^{*}=u\right\}}\left\{\beta_{u}^{-1} \mathbf{1}_{\left\{S_{u} \geq K\right\}}\left(y_{u} S_{u}-r_{u} K\right) d u-\frac{1}{2} \beta_{u}^{-1} \delta\left(S_{u}-K\right) d\langle S\rangle_{u}\right\} \mid \mathcal{F}_{t}\right] \\
= & \beta_{t} \mathbb{E}\left[\beta_{T}^{-1}\left(S_{T}-K\right)^{+} \mid \mathcal{F}_{t}\right]+\beta_{t} \mathbb{E}\left[\int_{\tau_{t}^{*}}^{T} \mathbf{1}_{\left\{\tau_{u}^{*}=u\right\}} \beta_{u}^{-1} \mathbf{1}_{\left\{S_{u} \geq K\right\}}\left(y_{u} S_{u}-r_{u} K\right) d u \mid \mathcal{F}_{t}\right],
\end{aligned}
$$

where, in the second equality, the last term vanishes due to the fact that the stock should be in-the-money region $\left(S_{u}>K\right)$ when the option is early exercised. It is now economically clear to see that the above result can be rewritten as

$$
V_{t}=\beta_{t} \mathbb{E}\left[\beta_{T}^{-1}\left(S_{T}-K\right)^{+} \mid \mathcal{F}_{t}\right]+\beta_{t} \mathbb{E}\left[\int_{t}^{T} \mathbf{1}_{\left\{V_{u} \leq\left(S_{u}-K\right)^{+}\right\}} \beta_{u}^{-1}\left(y_{u} S_{u}-r_{u} K\right) d u \mid \mathcal{F}_{t}\right] .
$$

Note that $\mathbf{1}_{\left\{V_{u} \leq\left(S_{u}-K\right)^{+}\right\}} \mathbf{1}_{\left\{S_{u}>K\right\}}=\mathbf{1}_{\left\{V_{u} \leq\left(S_{u}-K\right)^{+}\right\}}$since the option value should always be positive. For more rigorous treatment, see the related proof in [32, 23] as well as [2]. The case for a Put option can be shown similarly.

Now, from Eq. (2.2), one can see

$$
\beta_{t}^{-1} V_{t}+\int_{0}^{t} \beta_{u}^{-1} C_{u} \mathbf{1}_{\left\{V_{u} \leq \Psi^{+}\left(S_{u}\right)\right\}} d u
$$

is a $\mathbb{Q}$-martingale. Thus, we can conclude that the price of an American option satisfies

$$
\left\{\begin{array}{l}
d V_{t}=r_{t} V_{t} d t-C_{t} \mathbf{1}_{\left\{V_{t} \leq \Psi\left(S_{t}\right)\right\}} d t+Z_{t} \cdot d W_{t} \\
V_{T}=\Psi^{+}\left(S_{T}\right) \\
d S_{t}=\left(r_{t}-y_{t}\right) S_{t} d t+S_{t} \sigma_{t} \cdot d W_{t}, \quad S_{0}=s
\end{array}\right.
$$


where $Z \in \mathbb{R}^{d}$ is an appropriate $\mathcal{F}_{t}$-adapted process that should be solved at the same time with $V$. It is a non-linear FBSDE with a decoupled dynamics of forward component, or the stock process $S$. Here, we have replaced $\Psi^{+}$by $\Psi$ in the indicator function since $V$ should be clearly positive. In the next section, we carry out perturbative approximation procedures to solve the above FBSDE.

\section{Perturbative expansion and a particle method for FBSDE}

In [14], a systematic approximation procedures for a generic non-linear FBSDE is given. It treats the non-linear driver of the FBSDE as a perturbation and converted the original system into a series of decoupled linear FBSDEs, for which the issue is equivalent to solve general European contingent claims.

To apply the procedures, let us introduce perturbation parameter $\epsilon$ as

$$
\left\{\begin{array}{l}
d V_{t}^{(\epsilon)}=r_{t} V_{t}^{(\epsilon)} d t-\epsilon C_{t} \theta\left(\Psi\left(S_{t}\right)-V_{t}^{(\epsilon)}\right) d t+Z_{t}^{(\epsilon)} \cdot d W_{t} \\
V_{T}^{(\epsilon)}=\Psi^{+}\left(S_{T}\right)
\end{array}\right.
$$

where $\theta(\cdot)$ is the Heaviside step function. We now suppose that the solution of (3.1) can be expanded as a power series of $\epsilon$ :

$$
\begin{aligned}
& V_{t}^{(\epsilon)}=V_{t}^{(0)}+\epsilon V_{t}^{(1)}+\epsilon^{2} V_{t}^{(2)}+\epsilon^{3} V_{t}^{(3)}+\cdots \\
& Z_{t}^{(\epsilon)}=Z_{t}^{(0)}+\epsilon Z_{t}^{(1)}+\epsilon^{2} Z_{t}^{(2)}+\epsilon^{3} Z_{t}^{(3)}+\cdots .
\end{aligned}
$$

Economically speaking, we treat the early exercise premium as a perturbation and expand the price of American option around the corresponding European price. The method [14] allows to derive a series of linear FBSDEs specifying the dynamics of $\left(V^{(i)}, Z^{(i)}\right)_{i \geq 0}$ for each order of $\epsilon$. If the non-linear effects are sub-dominant and allow perturbative treatments, we can expect to obtain a reasonable approximation of the original model by setting $\epsilon=1$ at the end of the calculations. For the evaluation of an American option, the driver (or drift term) of the FBSDE is independent of the martingale component $Z$. Thus, in the following, we can focus on the level component $V$.

\subsection{0th order}

In the 0th order, we have

$$
\left\{\begin{array}{l}
d V_{t}^{(0)}=r_{t} V_{t}^{(0)} d t+Z_{t}^{(0)} \cdot d W_{t} \\
V_{T}^{(0)}=\Psi^{+}\left(S_{T}\right)
\end{array}\right.
$$

which clearly represents the dynamics of the corresponding European option price. We can easily see that it is solved as

$$
V_{t}^{(0)}=\beta_{t} \mathbb{E}_{t}\left[\beta_{T}^{-1} \Psi^{+}\left(S_{T}\right) \mid \mathcal{F}_{t}\right]
$$

Although there is no explicit expression of (3.3) for a generic stock process, it is always possible to obtain its approximation by asymptotic expansion (See [34, 25, 35, 36] for the 
details of asymptotic expansion.). It allows us, at least approximately, to have an explicit expression of $V_{t}^{(0)}$ as

$$
V_{t}^{(0)}=v^{(0)}\left(t, \mathbf{X}_{t}\right)
$$

where $\mathbf{X}_{t}=\left(S_{t}, r_{t}, y_{t}, \sigma_{t}, \cdots\right)$ contains all the relevant state processes. If necessary, application of Itô formula or using the process of Malliavin derivative $\left(\mathcal{D}_{t} X_{t}\right)$ yields the corresponding martingale component $Z^{(0)}$.

\subsection{1st order}

In the 1st order, the relevant FBSDE is given by

$$
\left\{\begin{array}{l}
d V_{t}^{(1)}=r_{t} V_{t}^{(1)} d t-C_{t} \theta\left(\Psi\left(S_{t}\right)-v^{(0)}\left(t, \mathbf{X}_{t}\right)\right) d t+Z_{t}^{(1)} \cdot d W_{t} \\
V_{T}^{(1)}=0
\end{array}\right.
$$

which is again linear and easy to integrate. We have

$$
V_{t}^{(1)}=\int_{t}^{T} d u \beta_{t} \mathbb{E}\left[\beta_{u}^{-1} C_{u} \theta\left(\Psi\left(S_{u}\right)-v_{u}^{(0)}\right) \mid \mathcal{F}_{t}\right]
$$

where, $v_{u}^{(0)}$ denotes $v^{(0)}\left(u, \mathbf{X}_{u}\right) . Z^{(1)}$ is obtained by the similar arguments given in the previous subsection. Although it is possible to evaluate (3.6) directly by Monte Carlo simulation, the time integration makes it rather time consuming. In fact, it soon becomes infeasible when one evaluates $\epsilon$-higher order expansion terms.

In order to avoid the difficulty, we adopt an interacting particle method proposed in Fujii \& Takahashi (2012d) [16]. We introduce an arbitrary $\mathcal{F}_{t}$-adapted strictly positive process $\left\{\lambda_{t}\right\}_{t \geq 0}$ to define

$$
\widehat{V}_{t, s}^{(1)}=\exp \left(\int_{t}^{s} \lambda_{u} d u\right) V_{s}^{(1)}
$$

and

$$
\widehat{C}_{t, s}=\frac{1}{\lambda_{s}} \exp \left(\int_{t}^{s} \lambda_{u} d u\right) C_{s}
$$

for $s \geq t$. Then, we have the SDE of $\widehat{V}_{t, s}^{(1)}$ for the time component $s(\geq t)$,

$$
\left\{\begin{array}{l}
d \widehat{V}_{t, s}^{(1)}=\left(r_{s}+\lambda_{s}\right) \widehat{V}_{t, s}^{(1)} d s-\lambda_{s} \widehat{C}_{t, s} \theta\left(\Psi\left(S_{s}\right)-v_{s}^{(0)}\right) d s+e^{\int_{t}^{s} \lambda_{u} d u} Z_{s}^{(1)} \cdot d W_{s} \\
\widehat{V}_{t, T}^{(1)}=0
\end{array} .\right.
$$

Since $\hat{V}_{t, t}^{(1)}=V_{t}^{(1)}$, we have

$$
\begin{aligned}
V_{t}^{(1)} & =\mathbb{E}\left[\int_{t}^{T} e^{-\int_{t}^{s}\left(r_{u}+\lambda_{u}\right) d u} \lambda_{s} \widehat{C}_{t, s} \theta\left(\Psi\left(S_{s}\right)-v_{s}^{(0)}\right) d s \mid \mathcal{F}_{t}\right] \\
& =\mathbf{1}_{\left\{\tau_{1}>t\right\}} \mathbb{E}\left[\mathbf{1}_{\left\{\tau_{1}<T\right\}} e^{-\int_{t}^{\tau_{1}} r_{u} d u} \widehat{C}_{t, \tau_{1}} \theta\left(\Psi\left(S_{\tau_{1}}\right)-v_{\tau_{1}}^{(0)}\right) \mid \mathcal{F}_{t}\right] .
\end{aligned}
$$

Here, $\tau_{1}$ is a $\mathcal{F}_{t}$-stopping time associated with the first jump of Poisson process whose intensity process is given by $\left\{\lambda_{t}\right\}_{t \geq 0}$. In contrast to (3.6), it is clear the expression of (3.11) 
allows one-shot Monte Carlo simulation. More detailed explanation for Monte Carlo simulation will be given in the later section. Although it is an interesting topic to obtain an optimal intensity process $\lambda$ that achieves the smallest variance in simulation, it is beyond the current scope of the paper. In the numerical examples, we simply use a constant intensity.

Remark: In [16], the intensity process $\lambda$ is assumed to be deterministic or an independent process for the other underlyings, which makes the evaluation of Malliavin derivatives required for $Z^{(i)}$ simpler. For the evaluation of American option, this assumption is not necessary since there is no need to obtain $Z^{(i)}$.

\subsection{2nd order}

For the 2nd order case, the relevant equation is given by

$$
\left\{\begin{array}{l}
d V_{t}^{(2)}=r_{t} V_{t}^{(2)} d t+C_{t} \delta\left(\Psi\left(S_{t}\right)-v_{t}^{(0)}\right) V_{t}^{(1)} d t+Z_{t}^{(2)} \cdot d W_{t} \\
V_{T}^{(2)}=0
\end{array}\right.
$$

where $\delta(\cdot)$ is a Dirac delta function as before. Since the FBSDE is linear, one can show easily that

$$
V_{t}^{(2)}=-\beta_{t} \int_{t}^{T} d u \mathbb{E}\left[\beta_{u}^{-1} C_{u} \delta\left(\Psi\left(S_{u}\right)-v_{u}^{(0)}\right) V_{u}^{(1)} \mid \mathcal{F}_{t}\right] .
$$

As mentioned in the previous section, the difficulty in a naive application of Monte Carlo simulation becomes much clearer now. At each point of time $u \in[t, T]$ in a given path, one needs the value of $V_{u}^{(1)}$, which in turn requires to run Monte Carlo simulation as well as time integration.

Therefore, let us define

$$
\widehat{V}_{t, s}^{(2)}=\exp \left(\int_{t}^{s} \lambda_{u} d u\right) V_{s}^{(2)}
$$

and use $\widehat{C}_{t, s}$ as before. Then, for $s \geq t$, we have

$$
d \widehat{V}_{t, s}^{(2)}=\left(r_{s}+\lambda_{s}\right) \widehat{V}_{t, s}^{(2)} d s+\lambda_{s} \widehat{C}_{t, s} \delta\left(\Phi\left(S_{s}\right)-v_{s}^{(0)}\right) V_{s}^{(1)} d s+e^{\int_{t}^{s} \lambda_{u} d u} Z_{s}^{(2)} \cdot d W_{s}
$$

with $\widehat{V}_{t, T}=0$. Thus, one obtains

$$
\begin{aligned}
& V_{t}^{(2)}=\widehat{V}_{t, t}^{(2)} \\
& =-\mathbb{E}\left[\int_{t}^{T} e^{-\int_{t}^{s}\left(r_{u}+\lambda_{u}\right) d u} \lambda_{s} \widehat{C}_{t, s} \delta\left(\Psi\left(S_{s}\right)-v_{s}^{(0)}\right) V_{s}^{(1)} d s \mid \mathcal{F}_{t}\right] \\
& =-\mathbf{1}_{\left\{\tau_{1}>t\right\}} \mathbb{E}\left[\mathbf{1}_{\left\{\tau_{1}<T\right\}} e^{-\int_{t}^{\tau_{1}} r_{u} d u} \widehat{C}_{t, \tau_{1}} \delta\left(\tau_{1}\right) V_{\tau_{1}}^{(1)} \mid \mathcal{F}_{t}\right] .
\end{aligned}
$$

Simple application of the tower property of iterated expectations gives

$$
V_{t}^{(2)}=-\mathbf{1}_{\left\{\tau_{1}>t\right\}} \mathbb{E}\left[\mathbf{1}_{\left\{\tau_{1}<\tau_{2}<T\right\}} e^{-\int_{t}^{\tau_{2}} r_{u} d u} \widehat{C}_{t, \tau_{1}} \delta\left(\tau_{1}\right) \widehat{C}_{\tau_{1}, \tau_{2}} \theta\left(\tau_{2}\right) \mid \mathcal{F}_{t}\right],
$$

where $\tau_{1}\left(\tau_{2}\right)$ is the first (second) jump time of the Poisson process with the intensity process $\lambda$. Here, we have written $\delta(\tau)=\delta\left(\Psi(\tau)-v_{\tau}^{(0)}\right)$ and $\theta(\tau)=\theta\left(\Psi(\tau)-v_{\tau}^{(0)}\right)$ to lighten the notations. 


\subsection{3 rd order}

In the 3rd order, the relevant dynamics becomes

$$
\left\{\begin{aligned}
d V_{t}^{(3)}=r_{t} V_{t}^{(3)} d t & +C_{t}\left\{\delta\left(\Psi\left(S_{t}\right)-v_{t}^{(0)}\right) V_{t}^{(2)}\right. \\
& \left.-\frac{1}{2} \partial \delta\left(\Psi\left(S_{t}\right)-v_{t}^{(0)}\right)\left(V_{t}^{(1)}\right)^{2}\right\} d t+Z_{t}^{(3)} \cdot d W_{t} \\
V_{T}^{(3)}=0 . &
\end{aligned}\right.
$$

Here, the derivative of a Dirac delta function can be evaluated by approximating the delta function as a normal density function with a small variance, or using the integration-byparts formula if possible. For the details of calculation, see the later sections treating numerical examples. After integration, we obtain

$$
\begin{aligned}
V_{t}^{(3)}= & -\beta_{t} \int_{t}^{T} d u \mathbb{E}\left[\beta_{u}^{-1} C_{u} \delta\left(\Psi\left(S_{u}\right)-V_{u}^{(0)}\right) V_{u}^{(2)} \mid \mathcal{F}_{t}\right] \\
& +\beta_{t} \int_{t}^{T} d u \mathbb{E}\left[\beta_{u}^{-1} C_{u} \frac{1}{2} \partial \delta\left(\Psi\left(S_{u}\right)-V_{u}^{(0)}\right)\left(V_{u}^{(1)}\right)^{2} \mid \mathcal{F}_{t}\right] .
\end{aligned}
$$

Let us compress a convoluted expectation as before. Let us denote

$$
\widehat{V}_{t, s}^{(3)}=\exp \left(\int_{t}^{s} \lambda_{u} d u\right) V_{s}^{(3)}
$$

and continue to use the simplified notations:

$$
\begin{aligned}
& \theta(t)=\theta\left(\Psi\left(S_{t}\right)-v_{t}^{(0)}\right) \\
& \delta(t)=\delta\left(\Psi\left(S_{t}\right)-v_{t}^{(0)}\right)
\end{aligned}
$$

Then, (3.18) is equivalent to

$$
d \widehat{V}_{t, s}^{(3)}=\left(r_{s}+\lambda_{s}\right) \widehat{V}_{t, s}^{(3)} d s+\lambda_{s} \widehat{C}_{t, s}\left\{\delta(s) V_{s}^{(2)}-\frac{1}{2} \partial \delta(s)\left[V_{s}^{(1)}\right]^{2}\right\} d s+e^{\int_{t}^{s} \lambda_{u} d u} Z_{s}^{(3)} \cdot d W_{s}
$$

with $\widehat{V}_{t, T}=0$, thus

$$
\begin{aligned}
& V_{t}^{(3)}=\mathbb{E}\left[\int_{t}^{T} e^{-\int_{t}^{s}\left(r_{u}+\lambda_{u}\right) d u} \lambda_{s} \widehat{C}_{t, s}\left\{-\delta(s) V_{s}^{(2)}+\frac{1}{2} \partial \delta(s)\left[V_{s}^{(1)}\right]^{2}\right\} d s \mid \mathcal{F}_{t}\right] \\
& =\mathbf{1}_{\left\{\tau_{1}>t\right\}} \mathbb{E}\left[\mathbf{1}_{\left\{\tau_{1}<T\right\}} e^{-\int_{t}^{\tau_{1}} r_{u} d u} \widehat{C}_{t, \tau_{1}}\left\{-\delta\left(\tau_{1}\right) V_{\tau_{1}}^{(2)}+\frac{1}{2} \partial \delta\left(\tau_{1}\right)\left[V_{\tau_{1}}^{(1)}\right]^{2}\right\} \mid \mathcal{F}_{t}\right] .
\end{aligned}
$$

Borrowing the idea from McKean [29] and use the tower property of iterated expectations, we finally obtain

$$
\begin{aligned}
& V_{t}^{(3)}=\mathbf{1}_{\left\{\tau_{1}>t\right\}} \mathbb{E}\left[\mathbf{1}_{\left\{\tau_{1}<\tau_{2}<\tau_{3}<T\right\}} e^{-\int_{t}^{\tau_{3}} r_{u} d u} \widehat{C}_{t, \tau_{1}} \delta\left(\tau_{1}\right) \widehat{C}_{\tau_{1}, \tau_{2}} \delta\left(\tau_{2}\right) \widehat{C}_{\tau_{2}, \tau_{3}} \theta\left(\tau_{3}\right) \mid \mathcal{F}_{t}\right] \\
& +\mathbf{1}_{\left\{\tau_{1}>t\right\}} \mathbb{E}\left[\mathbf{1}_{\left\{\tau_{1}<T\right\}} \frac{1}{2} e^{-\int_{t}^{\tau_{1}} r_{u} d u} \widehat{C}_{t, \tau_{1}} \partial \delta\left(\tau_{1}\right) \prod_{p=1}^{2}\left\{\mathbf{1}_{\left\{\tau_{1}<\tau_{2}^{(p)}<T\right\}} e^{-\int_{\tau_{1}}^{\tau_{2}^{(p)}} r_{u} d u} \widehat{C}_{\tau_{1}, \tau_{2}^{(p)}} \theta\left(\tau_{2}^{(p)}\right)\right\} \mid \mathcal{F}_{t}\right]
\end{aligned}
$$


with the $i$-th jump time of the Poisson process denoted by $\tau_{i}$.

In (3.23), $p=\{1,2\}$ indicates one of the two particle groups. In both of the groups, the relevant state variables (or particles) follow the common diffusion dynamics ( those specified by BS or Heston models, for example) but those belong to different groups are independent each other, i.e., driven by the two independent set of Brownian motions. This particle representation compresses $\left(\mathbb{E}\left[\cdot \mid \mathcal{F}_{\tau_{1}}\right]\right)^{2}$ into a single expectation operation.

More concretely, for the evaluation of the second line, we use the branching diffusion method of McKean. For a each path of simulation, we

(1): update the diffusion process of the underlyings $X=\{S, r, y, \sigma, \cdots\}$ in a standard way.

(2): do Poisson draw with intensity $\lambda$ at each step.

(3): if it draws a "jump" (or particles interact) at $\tau_{1}<T$, then the path yields the two identical copies of particles $\left\{\mathbf{X}_{p}\right\}_{p=1,2}$ of the underlying states as its offspring, which continue to evolve according to the identical diffusion equations but driven by the two independent set of Brownian motions.

(4): for each particle group, we continue the Poisson draw of the second interaction until the maturity.

(5): finally, extract the following term:

$$
\mathbf{1}_{\left\{\tau_{1}<T\right\}} \frac{1}{2} e^{-\int_{t}^{\tau_{1}} r_{u} d u} \widehat{C}_{t, \tau_{1}} \partial \delta\left(\tau_{1}\right) \prod_{p=1}^{2} \mathbf{1}_{\left\{\tau_{1}<\tau_{2}^{p}<T\right\}} e^{-\int_{\tau_{1}}^{\tau_{2}^{p}} r_{u} d u} \widehat{C}_{\tau_{1}, \tau_{2}^{p}} \theta\left(\tau_{2}^{p}\right)
$$

where $\tau_{2}^{p}$ is the second interaction time of each particle group.

(6): Repeat the procedures (1-5) and take the average of the values gathered in (5).

\subsection{4 th order}

We can continue the expansion to an arbitrary higher order. In the 4 th order, we have

$$
\left\{\begin{array}{cc}
d V_{t}^{(4)}=r_{t} V_{t}^{(4)} d t+C_{t}\left\{\frac{1}{3 !} \partial^{2} \delta\left(\Psi\left(S_{t}\right)-v_{t}^{(0)}\right)\left[V_{t}^{(1)}\right]^{3}-\partial \delta\left(\Psi\left(S_{t}\right)-v_{t}^{(0)}\right)\left[V_{t}^{(1)}\right]\left[V_{t}^{(2)}\right]\right. \\
\\
\left.+\delta\left(\Psi\left(S_{t}\right)-v_{t}^{(0)}\right)\left[V_{t}^{(3)}\right]\right\} d t+Z_{t}^{(4)} \cdot d W_{t} \\
V_{T}^{(4)}=0
\end{array}\right.
$$

and hence

$$
\begin{aligned}
V_{t}^{(4)}= & -\beta_{t} \int_{t}^{T} \mathbb{E}\left[\beta_{u}^{-1} \frac{1}{3 !} C_{u} \partial^{2} \delta\left(\Psi\left(S_{u}\right)-v_{u}^{(0)}\right)\left[V_{u}^{(1)}\right]^{3} \mid \mathcal{F}_{t}\right] d u \\
& +\beta_{t} \int_{t}^{T} \mathbb{E}\left[\beta_{u}^{-1} C_{u} \partial \delta\left(\Psi\left(S_{u}\right)-v_{u}^{(0)}\right)\left[V_{u}^{(1)}\right]\left[V_{u}^{(2)}\right] \mid \mathcal{F}_{t}\right] d u \\
& -\beta_{t} \int_{t}^{T} \mathbb{E}\left[\beta_{u}^{-1} C_{u} \delta\left(\Psi\left(S_{u}\right)-v_{u}^{(0)}\right) V_{u}^{(3)} \mid \mathcal{F}_{t}\right] d u
\end{aligned}
$$

Using the similar notations as in the previous sections, one can show that

$$
\begin{aligned}
d \widehat{V}_{t, s}^{(4)}= & \left(r_{s}+\lambda_{s}\right) \hat{V}_{t, s}^{(4)} d s+\lambda_{s} \widehat{C}_{t, s}\left\{\frac{1}{3 !} \partial^{2} \delta(s)\left[V_{s}^{(1)}\right]^{3}-\partial \delta(s)\left[V_{s}^{(1)}\right]\left[V_{s}^{(2)}\right]+\delta(s) V_{s}^{(3)}\right\} d s \\
& +e^{\int_{t}^{s} \lambda_{u} d u} Z_{s}^{(4)} \cdot d W_{s}
\end{aligned}
$$


and hence

$$
\begin{aligned}
& V_{t}^{(4)}=\mathbb{E}\left[\int_{t}^{T} e^{-\int_{t}^{s}\left(r_{u}+\lambda_{u}\right) d u} \lambda_{s} \widehat{C}_{t, s}\left\{-\frac{1}{3 !} \partial^{2} \delta(s)\left[V_{s}^{(1)}\right]^{3}+\partial \delta(s)\left[V_{s}^{(1)}\right]\left[V_{s}^{(2)}\right]-\delta(s)\left[V_{s}^{(3)}\right]\right\} d s \mid \mathcal{F}_{t}\right] \\
& =\mathbf{1}_{\left\{\tau_{1}>t\right\}} \mathbb{E}\left[\mathbf{1}_{\left\{\tau_{1}<T\right\}} e^{-\int_{t}^{\tau_{1}} r_{s} d s} \widehat{C}_{t, \tau_{1}}\left\{-\frac{1}{3 !} \partial^{2} \delta\left(\tau_{1}\right)\left[V_{\tau_{1}}^{(1)}\right]^{3}+\partial \delta\left(\tau_{1}\right)\left[V_{\tau_{1}}^{(1)}\right]\left[V_{\tau_{2}}^{(2)}\right]-\delta\left(\tau_{1}\right)\left[V_{\tau_{1}}^{(3)}\right]\right\} \mid \mathcal{F}_{t}\right]
\end{aligned}
$$

Using the tower property and particle representation, the above result can be expanded as

$$
\begin{aligned}
V_{t}^{(4)} & =-\mathbf{1}_{\left\{\tau_{1}>t\right\}} \mathbb{E}\left[\mathbf{1}_{\left\{\tau_{1}<T\right\}} \frac{1}{3 !} e^{-\int_{t}^{\tau_{1}} r_{s} d s} \widehat{C}_{t, \tau_{1}} \partial^{2} \delta\left(\tau_{1}\right) \prod_{p=1}^{3}\left\{\mathbf{1}_{\left\{\tau_{1}<\tau_{2}^{(p)}<T\right\}} e^{-\int_{\tau_{1}^{2}}^{\tau_{2}^{(p)}} r_{s} d s} \widehat{C}_{\tau_{1}, \tau_{2}^{(p)}} \theta\left(\tau_{2}^{(p)}\right)\right\}\right. \\
& +\mathbf{1}_{\left\{\tau_{1}<T\right\}} e^{-\int_{t}^{\tau_{1}} r_{s} d s} \widehat{C}_{t, \tau_{1}} \partial \delta\left(\tau_{1}\right)\left\{\mathbf{1}_{\left\{\tau_{1}<\tau_{2}^{(p)}<T\right\}} e^{-\int_{\tau_{1}}^{\tau_{2}^{(p)}} r_{s} d s} \widehat{C}_{\tau_{1}, \tau_{2}^{(p)}} \theta\left(\tau_{2}^{(p)}\right)\right\}^{p=1} \\
& \left.\times\left\{\mathbf{1}_{\left\{\tau_{1}<\tau_{2}^{(p)}<\tau_{3}^{(p)}<T\right\}} e^{-\int_{\tau_{1}^{3}}^{\tau_{3}^{(p)}} r_{s} d s} \widehat{C}_{\tau_{1}, \tau_{2}^{(p)}} \delta\left(\tau_{2}^{(p)}\right) \widehat{C}_{\tau_{2}^{(p)}, \tau_{3}^{(p)}} \theta\left(\tau_{3}^{(p)}\right)\right\}^{p=2}\right\}^{\tau_{4}} \widehat{C}_{\left\{\tau_{1}<\tau_{2}<\tau_{3}<\tau_{4}<T\right\}} e^{-\int_{t}^{\tau_{4}} r_{s} d s} \widehat{C}_{t, \tau_{1}} \delta\left(\tau_{1}\right) \widehat{C}_{\tau_{1}, \tau_{2}} \delta\left(\tau_{2}\right) \hat{C}_{\tau_{2}, \tau_{3}} \delta\left(\tau_{3}\right) \hat{C}_{\tau_{3}, \tau_{4}} \theta\left(\tau_{4}\right) \\
& +\mathbf{1}_{\left\{\tau_{1}<\tau_{2}<T\right\}} \frac{1}{2} e^{-\int_{t}^{\tau_{2}} r_{s} d s} \widehat{C}_{t, \tau_{1}} \delta\left(\tau_{1}\right) \widehat{C}_{\tau_{1}, \tau_{2}} \partial \delta\left(\tau_{2}\right) \\
& \left.\times \prod_{p=1}^{2}\left\{\mathbf{1}_{\left\{\tau_{2}<\tau_{3}^{(p)}<T\right\}} e^{-\int_{\tau_{2}}^{\tau_{3}^{(p)}} r_{s} d s} \widehat{C}_{\tau_{2}, \tau_{3}^{(p)}} \theta\left(\tau_{3}^{(p)}\right)\right\} \mid \mathcal{F}_{t}\right] .
\end{aligned}
$$

\section{Numerical Examples}

This section demonstrates the validity of our method proposed in the previous section through numerical experiments.

\subsection{Example 1: Black-Scholes model}

The first example is taken from Black-Scholes model:

$$
d S_{t} / S_{t}=(r-y) d t+\sigma d W_{t},
$$

where $r, y$ and $\sigma$ are all nonnegative constants. We calculate the values up to the fourth order terms based on our scheme derived as (3.11), (3.17), (3.23) and (3.27) with 10 million trials in Monte Carlo simulation. Here, we adopt the values reported in [22] as benchmarks. In particular, difficulty arises in differentiations up to the second order of the delta functions required for evaluation of the third (3.23) as well as the fourth (3.27) order terms. Since the density function in Black-Scholes model is explicitly known, we are able to apply integration by parts (IBP) for computation of these terms in order to avoid differentiation of the delta functions. Moreover, we approximate each delta function by a normal density function with mean zero and a certain variance, which enables direct evaluation of the expectation. 
Table 1: American Puts $(T=3, K=100, \sigma=0.2, r=0.08)$

\begin{tabular}{c|c|c|ccccc}
\hline & $S_{0}$ & Benchmark & 0th & 1 st & 2nd & 3rd & 4th \\
\hline$y=0.12$ & 80 & 25.658 & 24.777 & 25.829 & 25.854 & 25.799 & 25.739 \\
& 90 & 20.083 & 19.620 & 20.174 & 20.187 & 20.158 & 20.131 \\
& 100 & 15.498 & 15.252 & 15.546 & 15.553 & 15.538 & 15.518 \\
& 110 & 11.803 & 11.671 & 11.830 & 11.834 & 11.826 & 11.822 \\
& 120 & 8.886 & 8.814 & 8.900 & 8.902 & 8.897 & 8.894 \\
\hline$y=0.08$ & 80 & 22.205 & 19.525 & 23.553 & 22.847 & 22.265 & 22.194 \\
& 90 & 16.207 & 14.676 & 16.982 & 16.522 & 16.372 & 16.316 \\
& 100 & 11.704 & 10.817 & 12.151 & 11.885 & 11.800 & 11.735 \\
& 110 & 8.367 & 7.847 & 8.629 & 8.473 & 8.424 & 8.409 \\
& 120 & 5.930 & 5.622 & 6.081 & 5.989 & 5.962 & 5.951 \\
\hline$y=0.04$ & 80 & 20.350 & 14.589 & 23.683 & 22.236 & 21.450 & 20.348 \\
& 90 & 13.497 & 10.326 & 16.120 & 13.774 & 13.573 & 13.825 \\
& 100 & 8.944 & 7.168 & 10.390 & 9.132 & 9.070 & 8.706 \\
& 110 & 5.912 & 4.902 & 6.720 & 5.992 & 5.957 & 5.882 \\
& 120 & 3.898 & 3.315 & 4.360 & 3.953 & 3.928 & 3.827 \\
\hline$y=0.00$ & 80 & 20.000 & 10.253 & 24.338 & 22.044 & 20.892 & 20.063 \\
& 90 & 11.697 & 6.783 & 16.534 & 12.950 & 11.525 & 11.959 \\
& 100 & 6.932 & 4.406 & 9.590 & 6.719 & 7.004 & 6.697 \\
& 110 & 4.155 & 2.826 & 5.529 & 4.066 & 4.198 & 4.286 \\
& 120 & 2.510 & 1.797 & 3.232 & 2.457 & 2.506 & 2.582 \\
\hline
\end{tabular}

Number of simulations $=10,000,000, \lambda=2$, Number of time steps $=6000$

\begin{tabular}{c|c|c|ccccc}
\hline \multicolumn{7}{c}{ Error ratio } \\
\hline$y=0.12$ & $S_{0}$ & Benchmark & 0th & 1st & 2nd & 3rd & 4th \\
\hline & 90 & 25.658 & $-3.434 \%$ & $0.666 \%$ & $0.764 \%$ & $0.550 \%$ & $0.316 \%$ \\
& 90 & 20.083 & $-2.305 \%$ & $0.453 \%$ & $0.518 \%$ & $0.373 \%$ & $0.239 \%$ \\
& 100 & 15.498 & $-1.587 \%$ & $0.310 \%$ & $0.355 \%$ & $0.258 \%$ & $0.129 \%$ \\
& 110 & 11.803 & $-1.118 \%$ & $0.229 \%$ & $0.263 \%$ & $0.195 \%$ & $0.161 \%$ \\
& 120 & 8.886 & $-0.810 \%$ & $0.158 \%$ & $0.180 \%$ & $0.124 \%$ & $0.090 \%$ \\
\hline$y=0.08$ & 80 & 22.205 & $-12.069 \%$ & $6.071 \%$ & $2.891 \%$ & $0.270 \%$ & $-0.050 \%$ \\
& 90 & 16.207 & $-9.447 \%$ & $4.782 \%$ & $1.944 \%$ & $1.018 \%$ & $0.673 \%$ \\
& 100 & 11.704 & $-7.579 \%$ & $3.819 \%$ & $1.546 \%$ & $0.820 \%$ & $0.265 \%$ \\
& 110 & 8.367 & $-6.215 \%$ & $3.131 \%$ & $1.267 \%$ & $0.681 \%$ & $0.502 \%$ \\
& 120 & 5.93 & $-5.194 \%$ & $2.546 \%$ & $0.995 \%$ & $0.540 \%$ & $0.354 \%$ \\
\hline$y=0.04$ & 80 & 20.35 & $-28.310 \%$ & $16.378 \%$ & $9.268 \%$ & $5.405 \%$ & $-0.010 \%$ \\
& 90 & 13.497 & $-23.494 \%$ & $19.434 \%$ & $2.052 \%$ & $0.563 \%$ & $2.430 \%$ \\
& 100 & 8.944 & $-19.857 \%$ & $16.167 \%$ & $2.102 \%$ & $1.409 \%$ & $-2.661 \%$ \\
& 110 & 5.912 & $-17.084 \%$ & $13.667 \%$ & $1.353 \%$ & $0.761 \%$ & $-0.507 \%$ \\
& 120 & 3.898 & $-14.956 \%$ & $11.852 \%$ & $1.411 \%$ & $0.770 \%$ & $-1.821 \%$ \\
\hline$y=0.00$ & 80 & 20 & $-48.735 \%$ & $21.690 \%$ & $10.220 \%$ & $4.460 \%$ & $0.315 \%$ \\
& 90 & 11.697 & $-42.011 \%$ & $41.352 \%$ & $10.712 \%$ & $-1.470 \%$ & $2.240 \%$ \\
& 100 & 6.932 & $-36.440 \%$ & $38.344 \%$ & $-3.073 \%$ & $1.039 \%$ & $-3.390 \%$ \\
& 110 & 4.155 & $-31.986 \%$ & $33.069 \%$ & $-2.142 \%$ & $1.035 \%$ & $3.153 \%$ \\
& 120 & 2.51 & $-28.406 \%$ & $28.765 \%$ & $-2.112 \%$ & $-0.159 \%$ & $2.869 \%$ \\
\hline \multicolumn{7}{|c|}{ error ratio $=100 *($ value-benchmark) benchmark } &
\end{tabular}


Table 2: American Calls $(T=3, K=100)$

\begin{tabular}{l|c|c|ccccc}
\hline & $S_{0}$ & Benchmark & 0th & 1st & 2nd & 3rd & 4th \\
\hline$\sigma=0.2$ & 80 & 2.580 & 2.241 & 2.847 & 2.612 & 2.602 & 2.566 \\
$r=0.03$ & 90 & 5.167 & 4.355 & 5.822 & 5.240 & 5.199 & 5.140 \\
$y=0.07$ & 100 & 9.066 & 7.386 & 10.453 & 9.204 & 9.121 & 8.877 \\
& 110 & 14.443 & 11.331 & 17.036 & 14.763 & 14.566 & 14.322 \\
& 120 & 21.414 & 16.117 & 25.307 & 23.173 & 22.023 & 21.044 \\
\hline$\sigma=0.4$ & 80 & 11.326 & 10.309 & 11.998 & 11.475 & 11.399 & 11.373 \\
$r=0.03$ & 90 & 15.722 & 14.162 & 16.769 & 15.975 & 15.858 & 15.770 \\
$y=0.07$ & 100 & 20.793 & 18.532 & 22.318 & 21.132 & 20.948 & 20.851 \\
& 110 & 26.494 & 23.363 & 28.609 & 26.902 & 26.672 & 26.536 \\
& 120 & 32.781 & 28.598 & 35.599 & 33.390 & 33.000 & 32.681 \\
\hline$\sigma=0.3$ & 80 & 5.518 & 4.644 & 6.254 & 5.564 & 5.562 & 5.511 \\
$r=0.00$ & 90 & 8.842 & 7.269 & 10.197 & 8.951 & 8.914 & 8.763 \\
$y=0.07$ & 100 & 13.142 & 10.542 & 15.407 & 13.285 & 13.178 & 12.989 \\
& 110 & 18.453 & 14.430 & 22.004 & 18.655 & 18.726 & 18.144 \\
& 120 & 24.791 & 18.882 & 30.019 & 25.587 & 23.554 & 24.215 \\
\hline$\sigma=0.3$ & 80 & 12.146 & 12.133 & 12.148 & 12.148 & 12.147 & 12.146 \\
$r=0.07$ & 90 & 17.368 & 17.343 & 17.373 & 17.374 & 17.372 & 17.372 \\
$y=0.03$ & 100 & 23.348 & 23.301 & 23.359 & 23.360 & 23.357 & 23.355 \\
& 110 & 29.964 & 29.882 & 29.980 & 29.982 & 29.977 & 29.976 \\
& 120 & 37.104 & 36.972 & 37.130 & 37.134 & 37.125 & 37.120 \\
\hline
\end{tabular}

Number of simulations $=10,000,000, \lambda=2$, Number of time steps $=6000$

\begin{tabular}{l|c|c|ccccc}
\multicolumn{7}{c}{ Error ratio } \\
\hline$\sigma=0.2$ & $S_{0}$ & Benchmark & 0th & 1st & 2nd & 3rd & 4 th \\
$r=0.03$ & 90 & 5.167 & $-13.140 \%$ & $10.349 \%$ & $1.240 \%$ & $0.853 \%$ & $-0.543 \%$ \\
$y=0.07$ & 100 & 9.066 & $-18.531 \%$ & $15.299 \%$ & $1.522 \%$ & $0.607 \%$ & $-2.085 \%$ \\
& 110 & 14.443 & $-21.547 \%$ & $17.953 \%$ & $2.216 \%$ & $0.852 \%$ & $-0.838 \%$ \\
& 120 & 21.414 & $-24.736 \%$ & $18.180 \%$ & $8.214 \%$ & $2.844 \%$ & $-1.728 \%$ \\
\hline$\sigma=0.4$ & 80 & 11.326 & $-8.979 \%$ & $5.933 \%$ & $1.316 \%$ & $0.645 \%$ & $0.415 \%$ \\
$r=0.03$ & 90 & 15.722 & $-9.922 \%$ & $6.659 \%$ & $1.609 \%$ & $0.865 \%$ & $0.305 \%$ \\
$y=0.07$ & 100 & 20.793 & $-10.874 \%$ & $7.334 \%$ & $1.630 \%$ & $0.745 \%$ & $0.279 \%$ \\
& 110 & 26.494 & $-11.818 \%$ & $7.983 \%$ & $1.540 \%$ & $0.672 \%$ & $0.159 \%$ \\
& 120 & 32.781 & $-12.760 \%$ & $8.596 \%$ & $1.858 \%$ & $0.668 \%$ & $-0.305 \%$ \\
\hline$\sigma=0.3$ & 80 & 5.518 & $-15.839 \%$ & $13.338 \%$ & $0.834 \%$ & $0.797 \%$ & $-0.127 \%$ \\
$r=0.00$ & 90 & 8.842 & $-17.790 \%$ & $15.325 \%$ & $1.233 \%$ & $0.814 \%$ & $-0.893 \%$ \\
$y=0.07$ & 100 & 13.142 & $-19.784 \%$ & $17.235 \%$ & $1.088 \%$ & $0.274 \%$ & $-1.164 \%$ \\
& 110 & 18.453 & $-21.801 \%$ & $19.243 \%$ & $1.095 \%$ & $1.479 \%$ & $-1.675 \%$ \\
& 120 & 24.791 & $-23.835 \%$ & $21.088 \%$ & $3.211 \%$ & $-4.990 \%$ & $-2.323 \%$ \\
\hline$\sigma=0.3$ & 80 & 12.146 & $-0.107 \%$ & $0.016 \%$ & $0.016 \%$ & $0.008 \%$ & $0.000 \%$ \\
$r=0.07$ & 90 & 17.368 & $-0.144 \%$ & $0.029 \%$ & $0.035 \%$ & $0.023 \%$ & $0.023 \%$ \\
$y=0.03$ & 100 & 23.348 & $-0.201 \%$ & $0.047 \%$ & $0.051 \%$ & $0.039 \%$ & $0.030 \%$ \\
& 110 & 29.964 & $-0.274 \%$ & $0.053 \%$ & $0.060 \%$ & $0.043 \%$ & $0.040 \%$ \\
& 120 & 37.104 & $-0.356 \%$ & $0.070 \%$ & $0.081 \%$ & $0.057 \%$ & $0.043 \%$ \\
\hline \multicolumn{7}{|c|}{ error ratio $=100 *($ value-benchmark)/benchmark } &
\end{tabular}


Table 3: American Calls $(T=0.5, K=100, r=0.03, y=0.07)$

\begin{tabular}{c|c|c|ccccc}
\hline & $S_{0}$ & Benchmark & 0th & 1st & 2nd & 3rd & 4th \\
\hline$\sigma=0.2$ & 80 & 0.219 & 0.215 & 0.222 & 0.220 & 0.220 & 0.220 \\
& 90 & 1.386 & 1.345 & 1.413 & 1.391 & 1.389 & 1.389 \\
& 100 & 4.783 & 4.578 & 4.920 & 4.807 & 4.795 & 4.791 \\
& 110 & 11.098 & 10.421 & 11.569 & 11.172 & 11.137 & 11.221 \\
& 120 & 20.000 & 18.302 & 20.536 & 20.350 & 20.238 & 20.092 \\
\hline$\sigma=0.4$ & 80 & 2.689 & 2.651 & 2.710 & 2.695 & 2.692 & 2.693 \\
& 90 & 5.722 & 5.622 & 5.778 & 5.736 & 5.729 & 5.730 \\
& 100 & 10.239 & 10.021 & 10.365 & 10.272 & 10.257 & 10.261 \\
& 110 & 16.181 & 15.768 & 16.424 & 16.241 & 16.214 & 16.232 \\
& 120 & 23.360 & 22.650 & 23.778 & 23.459 & 23.411 & 23.395 \\
\hline
\end{tabular}

\begin{tabular}{|c|c|c|c|c|c|c|c|}
\hline \multicolumn{8}{|c|}{ Error ratio } \\
\hline & $S_{0}$ & Benchmark & 0th & $1 \mathrm{st}$ & 2nd & $3 \mathrm{rd}$ & 4 th \\
\hline \multirow[t]{5}{*}{$\sigma=0.2$} & 80 & 0.219 & $-1.826 \%$ & $1.370 \%$ & $0.457 \%$ & $0.457 \%$ & $0.457 \%$ \\
\hline & 90 & 1.386 & $-2.958 \%$ & $1.948 \%$ & $0.361 \%$ & $0.216 \%$ & $0.216 \%$ \\
\hline & 100 & 4.783 & $-4.286 \%$ & $2.864 \%$ & $0.502 \%$ & $0.251 \%$ & $0.167 \%$ \\
\hline & 110 & 11.098 & $-6.100 \%$ & $4.244 \%$ & $0.667 \%$ & $0.351 \%$ & $1.108 \%$ \\
\hline & 120 & 20 & $-8.490 \%$ & $2.680 \%$ & $1.750 \%$ & $1.190 \%$ & $0.460 \%$ \\
\hline \multirow[t]{5}{*}{$\sigma=0.4$} & 80 & 2.689 & $-1.413 \%$ & $0.781 \%$ & $0.223 \%$ & $0.112 \%$ & $0.149 \%$ \\
\hline & 90 & 5.722 & $-1.748 \%$ & $0.979 \%$ & $0.245 \%$ & $0.122 \%$ & $0.140 \%$ \\
\hline & 100 & 10.239 & $-2.129 \%$ & $1.231 \%$ & $0.322 \%$ & $0.176 \%$ & $0.215 \%$ \\
\hline & 110 & 16.181 & $-2.552 \%$ & $1.502 \%$ & $0.371 \%$ & $0.204 \%$ & $0.315 \%$ \\
\hline & 120 & 23.36 & $-3.039 \%$ & $1.789 \%$ & $0.424 \%$ & $0.218 \%$ & $0.150 \%$ \\
\hline
\end{tabular}

As for the third order term, consulting the results based on IBP, we are capable of determining an appropriate size of the variance for each normal density applied in the approximation of a delta function. Unfortunately, however, this IBP method does not yield stable results for some cases in computing the fourth-order term. It is clear that we want to use a small enough variance for the normal density so that it is a reasonable approximation of the delta function. On the other hand, too small variance increases the variation (dispersion) of simulation result. Therefore, we change the variance from some large value to a smaller one gradually for a given number of simulation paths and picks up the smallest value beyond which the variation (dispersion) starts to increase.

This scheme can be applied to general cases where the density functions of the underlying models are not explicitly available. In fact, we adopt this approach for the numerical example for the Heston model in the next subsection 3. Of course, there is no guarantee that the choice of variance that gives the smallest dispersion in simulation also yields the smallest bias in the numerical result. However, the numerical result suggests that the method produces accurate enough approximation for practical use given a reasonable

\footnotetext{
${ }^{3}$ Notice that there is no need to use unnecessarily small variance for the approximation of delta function. Intuitively speaking, the delta function within the expectation operation extracts the density where its argument vanishes. Thus, as long as the density functions of the underlyings do not change significantly within a given range, one can use it as a variance of the normal density function as an approximation of the corresponding delta function.
} 
number of simulation paths. Note here that the large number of paths used in this example is to confirm the convergence of higher order expansions. For practical pricing purpose, as can be seen in the following example of Heston model, there is no need to run such a large number of simulation trials.

Table 1 presents the result for American put options with $T=3, K=100, \sigma=0.2$ and $r=0.08$, which confirms that the error ratios become improved in the results up to the third or the fourth order comparing with those up to the first and the second orders. In total, the approximations up to the fourth order provide the most precise ones in terms of the error ratios. Note also that for the dividend rate $y=0.12$ and 0.07 , adding the fourth order term to the third one makes the accuracies of the approximations improved, while for $y=0.04$ and 0.00 , it makes the accuracies worse in three and four out of the five cases, respectively. Table $2(T=3, K=100)$ and Table $3(T=0.5, K=100, r=0.03, y=0.07)$ present the results for American call options, which shows the error ratios become smaller by adding the third or/and fourth order terms.

\subsection{Example 2: Heston model}

The next example takes Heston model (4.2):

$$
\begin{aligned}
d S_{t} & =(r-y) S_{t} d t+S_{t} \sqrt{\nu_{t}} \cdot d W_{1, t} \\
d \nu_{t} & =\xi\left(\theta-\nu_{t}\right) d t+\eta \sqrt{\nu_{t}}\left(\rho d W_{1, t}+\sqrt{1-\rho^{2}} d W_{2, t}\right) ; \quad \nu_{0}=\sigma^{2},
\end{aligned}
$$

where $\xi, \eta$ and $\theta$ are positive constants such that $\xi \eta \geq \theta^{2} / 2$ and $W_{1, t} \perp W_{2, t}$. Then, we compute the approximate values for American put prices $(T=0.25,0.5, K=100$, $r=0.05, y=0, \eta=0.1, \xi=3.0, \theta=0.04)$ up to the third order based on our scheme, (3.11), (3.17) and (3.23) with 50,000 trials in Monte Carlo simulation. Here, we adopt [1] as the benchmark values, in which a two-dimensional tree with two hundred time steps and a Control Variate technique is applied. Moreover, an asymptotic expansion method, particularly, the equation appearing in p.113 of [36] is used for computation of European option prices, that is, 0 -th order $v^{(0)}$.

Table 4 demonstrates that our method works effectively in the Heston model, which suggests its applicability to the pricing problem of American options for other multidimensional models, too. The numerical result shows that the expansion up to the third order improves the accuracies in most of the cases. For the choice of the normal density as a approximate delta function, we have used the variance found to work well in the previous BS model. In the case where it produces too much dispersion in simulation, we have applied the general methodology explained in the previous subsection to pick up an appropriate variance. The example shows that the relatively small number of simulation trials is enough to obtain a reasonable accuracy for the practical use. In Table 5, we have given the numerical results with larger number of simulations 500,000 for the same set of American options with $T=0.5$ in Table 4, Although the improvement of accuracy from the second to the third order approximation becomes more robust in this case, one can observe that the size of the change in option prices is rather small.

\section{Remark:}

Although higher order integration is required, the direct evaluation of (3.13) ( and corresponding expressions in other orders ) is also possible once we know the transition density 
of the underlying states. For the diffusion models, it is always possible to obtain approximation using the asymptotic expansion [34]. If there exists efficient enough integration technique, such as Gaussian quadrature and its extension, it could provide another pricing technique. In fact, in BS model, we have compared this semi-analytic results (by brute force integration within \pm 5 -sigma range and using a normal density function with variance of $1 \mathrm{bp}$ of the stock process at each time as an approximation for the delta function) to those obtained from the particle method up to the second order terms. We confirmed the consistency between their numerical results.

Table 4: American Puts in Heston model $(K=100, r=0.05, y=0, \eta=0.1, \xi=3.0, \theta=$ $0.04)$

\begin{tabular}{l|c|c|cccc|cccc}
\hline & $S_{0}$ & Benchmark & Oth & 1st & 2nd & 3rd & ER(0th) & ER(1st) & ER(2nd) & ER(3rd) \\
\hline$\rho=-0.1$ & 90 & 10.171 & 9.643 & 10.507 & 10.293 & 10.141 & $-5.18 \%$ & $3.31 \%$ & $1.20 \%$ & $-0.29 \%$ \\
$\sigma=0.2$ & 100 & 3.475 & 3.374 & 3.556 & 3.486 & 3.481 & $-2.89 \%$ & $2.35 \%$ & $0.34 \%$ & $0.19 \%$ \\
& 110 & 0.774 & 0.758 & 0.783 & 0.775 & 0.775 & $-2.03 \%$ & $1.21 \%$ & $0.18 \%$ & $0.14 \%$ \\
\hline$\rho=-0.7$ & 90 & 10.121 & 9.573 & 10.455 & 10.253 & 10.101 & $-5.41 \%$ & $3.31 \%$ & $1.31 \%$ & $-0.19 \%$ \\
$\sigma=0.2$ & 100 & 3.481 & 3.383 & 3.559 & 3.493 & 3.487 & $-2.81 \%$ & $2.24 \%$ & $0.36 \%$ & $0.18 \%$ \\
& 110 & 0.842 & 0.829 & 0.854 & 0.845 & 0.845 & $-1.53 \%$ & $1.43 \%$ & $0.37 \%$ & $0.41 \%$ \\
\hline$\rho=-0.1$ & 90 & 12.182 & 11.896 & 12.347 & 12.190 & 12.173 & $-2.35 \%$ & $1.36 \%$ & $0.07 \%$ & $-0.08 \%$ \\
$\sigma=0.4$ & 100 & 6.496 & 6.379 & 6.572 & 6.504 & 6.501 & $-1.80 \%$ & $1.17 \%$ & $0.12 \%$ & $0.08 \%$ \\
& 110 & 3.091 & 3.047 & 3.118 & 3.092 & 3.092 & $-1.43 \%$ & $0.85 \%$ & $0.03 \%$ & $0.02 \%$ \\
\hline$\rho=-0.7$ & 90 & 12.112 & 11.832 & 12.291 & 12.132 & 12.116 & $-2.31 \%$ & $1.47 \%$ & $0.16 \%$ & $0.03 \%$ \\
$\sigma=0.4$ & 100 & 6.490 & 6.377 & 6.565 & 6.505 & 6.503 & $-1.74 \%$ & $1.16 \%$ & $0.23 \%$ & $0.20 \%$ \\
& 110 & 3.146 & 3.104 & 3.180 & 3.157 & 3.157 & $-1.31 \%$ & $1.11 \%$ & $0.36 \%$ & $0.37 \%$ \\
\hline
\end{tabular}
Number of simulations $=50,000, \lambda=4$, Number of time steps $=2000$

$\mathrm{ER}=100 *($ value-benchmark $) /$ benchmark

\begin{tabular}{|c|c|c|c|c|c|c|c|c|c|c|}
\hline \multicolumn{11}{|c|}{$T=0.5$} \\
\hline & $S_{0}$ & Benchmark & Oth & $1 \mathrm{st}$ & 2nd & $3 \mathrm{rd}$ & $\mathrm{ER}(0 \mathrm{th})$ & $\mathrm{ER}(1 \mathrm{st})$ & $\mathrm{ER}(2 \mathrm{nd})$ & $\mathrm{ER}(3 \mathrm{rd})$ \\
\hline$\rho=-0.1$ & 90 & 10.648 & 9.864 & 11.236 & 10.752 & 10.532 & $-7.36 \%$ & $5.53 \%$ & $0.98 \%$ & $1.09 \%$ \\
\hline \multirow{2}{*}{$\sigma=0.2$} & 100 & 4.647 & 4.423 & 4.835 & 4.676 & 4.665 & $-4.83 \%$ & $4.04 \%$ & $0.61 \%$ & $0.38 \%$ \\
\hline & 110 & 1.683 & 1.624 & 1.733 & 1.692 & 1.693 & $-3.50 \%$ & $2.94 \%$ & $0.55 \%$ & $0.55 \%$ \\
\hline \multirow{3}{*}{$\begin{array}{l}\rho=-0.7 \\
\sigma=0.2\end{array}$} & 90 & 10.564 & 9.766 & 11.183 & 10.688 & 10.490 & $-7.55 \%$ & $5.87 \%$ & $1.18 \%$ & $0.70 \%$ \\
\hline & 100 & 4.664 & 4.443 & 4.844 & 4.684 & 4.678 & $-4.73 \%$ & $3.88 \%$ & $0.43 \%$ & $0.31 \%$ \\
\hline & 110 & 1.787 & 1.732 & 1.837 & 1.798 & 1.797 & $-3.08 \%$ & $2.79 \%$ & $0.61 \%$ & $0.52 \%$ \\
\hline \multirow{3}{*}{$\begin{array}{l}\rho=-0.1 \\
\sigma=0.4\end{array}$} & 90 & 13.314 & 12.712 & 13.664 & 13.375 & 13.283 & $-4.52 \%$ & $2.63 \%$ & $0.46 \%$ & $0.23 \%$ \\
\hline & 100 & 8.008 & 7.705 & 8.207 & 8.070 & 8.021 & $-3.78 \%$ & $2.48 \%$ & $0.77 \%$ & $0.16 \%$ \\
\hline & 110 & 4.545 & 4.399 & 4.642 & 4.567 & 4.550 & $-3.21 \%$ & $2.12 \%$ & $0.48 \%$ & $0.09 \%$ \\
\hline \multirow{3}{*}{$\begin{array}{l}\rho=-0.7 \\
\sigma=0.4\end{array}$} & 90 & 13.217 & 12.625 & 13.602 & 13.314 & 13.229 & $-4.48 \%$ & $2.91 \%$ & $0.73 \%$ & $0.09 \%$ \\
\hline & 100 & 8.000 & 7.705 & 8.196 & 8.048 & 8.012 & $-3.69 \%$ & $2.46 \%$ & $0.60 \%$ & $0.15 \%$ \\
\hline & 110 & 4.620 & 4.479 & 4.709 & 4.627 & 4.612 & $-3.04 \%$ & $1.93 \%$ & $0.16 \%$ & $0.17 \%$ \\
\hline
\end{tabular}

$\mathrm{ER}=100 *($ value-benchmark $) /$ benchmark

\section{Conclusions}

This paper proposed a new calculation technique for American options in an FBSDE framework. The well-known decomposition of an American option price with that of the corresponding European option and additional early exercise premium can be written in a form of a decoupled non-linear FBSDE. We have used the recently proposed perturbation technique of FBSDE with an interacting particle method to obtain numerical results. We have tested the effectiveness of our approximation by comparing the numerical results 
Table 5: The same setup with $T=0.5$ in Table 4 but using larger number of simulation.

\begin{tabular}{l|c|c|cccc|cccc}
\hline & $S_{0}$ & Benchmark & 0th & 1 st & 2nd & 3rd & ER(0th) & ER(1st) & ER(2nd) & ER(3rd) \\
\hline$\rho=-0.1$ & 90 & 10.648 & 9.864 & 11.259 & 10.758 & 10.540 & $-7.36 \%$ & $5.74 \%$ & $1.03 \%$ & $-1.01 \%$ \\
$\sigma=0.2$ & 100 & 4.647 & 4.423 & 4.831 & 4.674 & 4.653 & $-4.83 \%$ & $3.95 \%$ & $0.57 \%$ & $0.11 \%$ \\
& 110 & 1.683 & 1.624 & 1.729 & 1.688 & 1.683 & $-3.50 \%$ & $2.70 \%$ & $0.28 \%$ & $-0.02 \%$ \\
\hline$\rho=-0.7$ & 90 & 10.564 & 9.766 & 11.173 & 10.696 & 10.457 & $-7.55 \%$ & $5.77 \%$ & $1.26 \%$ & $-1.01 \%$ \\
$\sigma=0.2$ & 100 & 4.664 & 4.443 & 4.841 & 4.688 & 4.672 & $-4.73 \%$ & $3.81 \%$ & $0.53 \%$ & $0.19 \%$ \\
& 110 & 1.787 & 1.732 & 1.839 & 1.800 & 1.795 & $-3.08 \%$ & $2.86 \%$ & $0.71 \%$ & $0.44 \%$ \\
\hline$\rho=-0.1$ & 90 & 13.314 & 12.712 & 13.676 & 13.384 & 13.311 & $-4.52 \%$ & $2.72 \%$ & $0.53 \%$ & $-0.02 \%$ \\
$\sigma=0.4$ & 100 & 8.008 & 7.705 & 8.202 & 8.043 & 8.007 & $-3.78 \%$ & $2.41 \%$ & $0.44 \%$ & $-0.01 \%$ \\
& 110 & 4.545 & 4.399 & 4.643 & 4.561 & 4.546 & $-3.21 \%$ & $2.14 \%$ & $0.34 \%$ & $0.02 \%$ \\
\hline$\rho=-0.7$ & 90 & 13.217 & 12.625 & 13.582 & 13.292 & 13.216 & $-4.48 \%$ & $2.76 \%$ & $0.57 \%$ & $-0.01 \%$ \\
$\sigma=0.4$ & 100 & 8.000 & 7.705 & 8.194 & 8.039 & 8.003 & $-3.69 \%$ & $2.42 \%$ & $0.49 \%$ & $0.04 \%$ \\
& 110 & 4.620 & 4.479 & 4.718 & 4.640 & 4.625 & $-3.04 \%$ & $2.12 \%$ & $0.43 \%$ & $0.10 \%$ \\
\hline \multicolumn{7}{c}{ Number of simulations = 500,000, $\lambda=8$, Number of time steps $=2000$} & &
\end{tabular}

to those obtained from existing tree algorithms. Although there remains some subtlety for choosing an appropriate variance for the normal density function as a proxy of the Dirac delta function, the proposed method for the variance choice yields accurate enough approximations for BS as well as Heston models. In the paper, we could only test a narrow range of parameters with relatively short expiries of options due to the limitation of existing benchmark results. However, the results are quite encouraging to suggest that our perturbation technique combined with an interacting particle method can be applied to much broader range of models and parameters.

\section{References}

[1] Beliaeva, A. N. and Nawalkha, K. S. (2010), "A Simple Approach to Pricing American options under the Heston Stochastic Volatility Model", available at http://ssrn.com/abstract=1107934.

[2] Benth, F., Karlsen, K, Reikvam, K. (2003), "A semilinear Black and Scholes partial differential equation for valuing American options," Finance and Stochastics 7: 277298.

[3] Bielecki, T. and Rutkowski, M. (2002), "Credit Risk: Modeling, Valuation and Hedging," Springer Finance.

[4] Bielecki, T., Jeanblanc, M. and Rutkowski, M. (2009), "Credit Risk Modeling," Osaka University Press.

[5] Bismut, J.M. (1973), "Conjugate Convex Functions in Optimal Stochastic Control," J. Political Econ., 3, 637-654.

[6] Carmona (editor) (2009), "Indifference Pricing," Princeton University Press.

[7] Carr, P., Jarrow, R., Myneni, R. (1992), "Alternative characterizations of American put option," Math. Finance 2:87-106. 
[8] Crépey, S. (2012), "A BSDE Approach to Counterparty Risk under Funding Constraints-I (II)". Forthcoming in Mathematical Finance.

[9] Cvitanić, J. and Zhang, J. (2012), "Contract Theory in Continuous-Time Models," Springer Finance.

[10] Duffie, D., Huang, M. (1996), "Swap Rates and Credit Quality," Journal of Finance, Vol. 51, No. 3, 921.

[11] El Karoui, N., Peng, S.G., and Quenez, M.C. (1997a), "Backward stochastic differential equations in finance," Math. Finance 7 1-71.

[12] Fujita, H., "On the blowing up of solutions of the Cauchy problem for $u_{t}=\Delta u+$ $u^{1+\alpha}, " J$. Fac. Sci. Univ. Tokyo 13 (1966), 109-124.

[13] Fujii, M. and Takahashi, A. (2012a), "Derivative Pricing under Asymmetric and Imperfect Collateralization and CVA," Forthcoming in Quantitative Finance.

[14] Fujii, M. and Takahashi, A. (2012b), "Analytical approximation for non-linear FBSDEs with perturbation scheme," International Journal of Theoretical and Applied Finance, Vol.15, Issue 05, 1250034(24).

[15] Fujii, M. and Takahashi, A. (2012c), "Perturbative Expansion of FBSDE in an Incomplete Market with Stochastic Volatility," Quarterly Journal of Finance, Vol. 2, No. 31250015 (24).

[16] Fujii, M. and Takahashi, A. (2012d), "Perturbative Expansion Technique for NonLinear FBSDEs with Interacting Particle Method," CARF working paper series, CARF-F-278.

[17] Ikeda, N., Nagasawa, M., and Watanabe, S. (1965), "Branching Markov processes," Abstracts Proc. Japan Acad. 41, 816-821.

[18] Ikeda, N., Nagasawa, M., and Watanabe, S. (1966), "Branching Markov processes," Abstracts Proc. Japan Acad. 42, 252-257, 370-375, 380-384, 719-724, 1016-1021, 10221026 .

[19] Ikeda, N., Nagasawa, M., and Watanabe, S., (1968), "Branching Markov processes I(II)," J. Math. Kyoto Univ. 8, 233-278, 365-410.

[20] Ikeda, N. et.al., (1966, 1967) hSeminar on Probability,h vol:23 I-II and vol:25 I-II. (in Japanese.)

[21] Jacka, S. D., (1991), "Optimal stopping and the American put," Math. Finance 1:114.

[22] Ju, N., Zhong, R. (1999), "An approximate formula for pricing American options," Journal of Derivatives.

[23] Karatzas, I., Shreve, S. (1998), "Methods of Mathematical Finance," Springer-Verlag. 
[24] Kim, I. J., (1990), "The analytical valuation of American options," Rev. Financial Studies 3: 547-572.

[25] Kunitomo, N. and Takahashi, A. (2003), "On Validity of the Asymptotic Expansion Approach in Contingent Claim Analysis," Annals of Applied Probability, 13, No.3, 914-952.

[26] Henry-Labordère, P. (2012), "Counterparty Risk Valuation: A marked branching diffusion approach", arXiv:1203.2369

[27] Ma, J., Protter, P., and Yong, J. (1994), "Solving forward-backward stochastic differential equations explicitly", Prob.\& Related Fields, 98.

[28] Ma, J., and Yong, J. (2000), "Forward-Backward Stochastic Differential Equations and their Applications," Springer.

[29] McKean, H., P. (1975), "Application of Brownian Motion to the Equation of Kolmogorov-Petrovskii-Piskunov," Communications on Pure and Applied Mathematics, Vol. XXVIII, 323-331.

[30] Nagasawa, M., Sirao, T., (1969), "Probabilistic Treatment of the Blowing up of Solutions for a Nonlinear Integral Equation," Trans. Amer. Math. Soc. Vol 139, 301-310.

[31] Pardoux, E., and Peng, S. (1990), "Adapted Solution of a Backward Stochastic Differential Equation," Systems Control Lett., 14, 55-61.

[32] Rutkowski, M. (1994), "The early exercise premium representation of foreign market American options," Math. Finance 4 (4):313-325.

[33] Saito, T. and Takahashi, A.(2004), "Pricing American Option -an Asymptotic Expansion Approach-," Monetary and Economic Studies 22(2):35-88. (in Japanese.)

[34] Takahashi, A. (1999), "An Asymptotic Expansion Approach to Pricing Contingent Claims," Asia-Pacific Financial Markets, 6, 115-151.

[35] Takahashi, A., Takehara, K., and Toda, M. (2011), "A General Computation Scheme for a High-Order Asymptotic Expansion Method," CARF Working Paper F-242, available at http://www.carf.e.u-tokyo.ac.jp/workingpaper/.

[36] Takahashi, A. and Yamada, T. (2012a), "An Asymptotic Expansion with Push-Down of Malliavin Weights," SIAM Journal on Financial Mathematics, Volume 3, pp 95136.

[37] Takahashi, A., and Yamada, T. (2012b), "An Asymptotic Expansion for ForwardBackward SDEs: A Malliavin Calculus Approach," CARF Working paper series, CARF-F-296. 